\title{
The cost-effectiveness implications of suboptimal treatment for different severities of Alzheimer's disease in the UK
}

\author{
Darshan Zala ${ }^{1}$ D, Dennis Chan ${ }^{2}$ and Paul McCrone ${ }^{1}$ \\ ${ }^{1}$ Department of Health Service and Population Research, Institute of Psychiatry, Psychology and Neuroscience, King's College London, \\ London, UK \\ ${ }^{2}$ Department of Clinical Neurosciences, University of Cambridge, Cambridge, UK \\ Correspondence to: D. Zala, E-mail: darshanzala@hotmail.co.uk
}

\begin{abstract}
Objective: This study aims to evaluate the impact of suboptimal treatment, defined in terms of lower population coverage (percentage of total patient population receiving optimal treatment) and delay to treatment on the cost-effectiveness of pharmacological therapies approved for the treatment of different severities of Alzheimer's disease (AD) in the UK.
\end{abstract}

Methods: A 5-year Markov model was used to simulate transition to full-time care, as delay and coverage were varied for $\mathrm{AD}$ patients with mild-to-moderate and moderate-to-severe dementia. The timevarying predictive equations, resource use, utilities, treatment effects and mortality were derived using published sources.

Results: For the cohort with moderate-to-severe dementia, cost-effectiveness was optimised when delay was minimised and coverage maximised. For mild-to-moderate dementia, results were similar but varied widely depending on the inputted cost of acetylcholinesterase inhibitors.

Conclusions: The average cost-effectiveness of pharmacological treatments for AD is sensitive to delays to treatment and population coverage. The results of this study can inform future healthcare policy in order to maximise cost-effectiveness of pharmacological therapies for AD. Copyright (C) 2017 John Wiley \& Sons, Ltd. Key words: acetylcholinesterase inhibitors; memantine; Alzheimer's disease; cost-effectiveness; delay to treatment; early
assessment; treatment coverage

History: Received 31 January 2017; Accepted 19 April 2017; Published online 14 June 2017 in Wiley Online Library (wileyonlinelibrary.com)

DOI: 10.1002 /gps.4745

\section{Introduction}

Alzheimer's disease (AD) accounts for $60-80 \%$ of all cases of dementia in the UK (Alzheimer's Association, 2011). At present, the number of individuals with dementia is estimated to be around 700000 (Prince et al., 2014), and, in line with the ageing population, this figure is calculated to rise to 1.5 million by 2050 . This rise in numbers will be associated with a steep rise in the social and economic costs borne by patients, their caregivers and health and social care services.

The UK National Institute for Health and Care Excellence (NICE) has approved acetylcholinesterase inhibitors (AChEIs) for all severities of $\mathrm{AD}$ and the N-Methyl-D-aspartate (NMDA) receptor antagonist memantine for the treatment of moderate-to-severe AD (NICE, 2016). These decisions were based on a review of clinical and economic evidence (NICE, 2011). Most economic evaluations have indicated that these pharmacological treatments are cost-effective (Green et al., 2005; Green, 2007; Cohen and Neumann, 2008; Rive et al., 2010a), primarily as a result of their effect in delaying the requirement for full-time care (FTC) with its attendant high costs.

Studies have suggested that cost-effectiveness is sensitive to early diagnosis and initiation of therapy (Barnett et al., 2014; Banerjee and Wittenberg, 2009; Weimer and Sager, 2009; Getsios et al., 2012), but there has been little research on the effects of coverage. As a result of delays to diagnosis and low rates of diagnosis, 
the proportion of undiagnosed $\mathrm{AD}$ cases has been estimated to be at least 50\% (Weimer and Sager, 2009; National Audit Office, 2007), and the mean delay from onset of symptoms to diagnosis in the UK has been estimated to be around 36 months (Bond et al., 2005).

This analysis aims to assess the cost-effectiveness implications of the suboptimal treatment of $\mathrm{AD}$ in the UK. Suboptimal is defined as any divergence from $100 \%$ treatment coverage and/or from immediate implementation of NICE recommended pharmacological therapy from the time of diagnosis. Suboptimal treatment can occur as a result of failure to diagnose affected individuals, attribution of incorrect diagnosis to affected individuals (e.g. diagnosis of vascular dementia instead of $\mathrm{AD}$ ) and failure to initiate treatment at the time of diagnosis.

This study differs from previous work in several ways. First, the model uses already available treatments, rather than hypothetical treatments, in contrast to other analyses (Barnett et al., 2014). Second, because it does not explicitly model a hypothetical 'real world' policy in contrast to other evaluations (Getsios et al., 2012), it allows exploration of a wider variety of scenarios in terms of treatment coverage and delay. Third, the model investigates cost-effectiveness for differing severities of $\mathrm{AD}$ dementia. While this study does not directly investigate the service costs associated with increasing treatment coverage and reducing treatment delay, we illustrate how these costs can be integrated with the analysis using examples.

\section{Methods}

\section{Original model}

The model used in this analysis is an adaptation of the Markov model used in the successful NICE submission of memantine for moderate-to-severe $\mathrm{AD}$ in the $\mathrm{UK}$
(Rive et al., 2010a; Rive et al., 2010b). This included replicating the model for a mild-to-moderate cohort, by replacing key parameters. The definition of FTC was based on location of care and an assessment of physical and functional disability (Rive et al., 2010a). A UK cohort of $\mathrm{AD}$ patients, with moderate-to-severe dementia, started in pre-FTC and could either remain in this state or transition each month (Sonnenberg and Beck, 1993), from pre-FTC to FTC or from pre-FTC to death.

\section{Populations and treatments}

In view of the differing NICE recommended treatments for differing $\mathrm{AD}$ severity, the current study modelled two populations: (i) moderate-to-severe $\mathrm{AD}$ dementia, 'optimal' treatment memantine being compared with 'suboptimal' treatment (AChEIs alone or no treatment); (ii) mild-to-moderate $\mathrm{AD}$ dementia, 'optimal' treatment (AChEIs) compared with 'suboptimal' treatment (no AChEIs).

\section{Adaptations to original model}

Two main adaptations were made to the Rive et al., 2010a model for memantine (Figure 1). First, the 5 -year time horizon was divided into a suboptimal treatment phase, characterised by $\leq 100 \%$ treatment coverage and/or $\geq 0$ years of delay to optimal treatment, followed by an optimal treatment phase where all patients receive the NICE recommended treatment for their AD severity (1A). Second, the pre-FTC state was split in two: those receiving optimal treatment from the beginning and those receiving suboptimal treatment until the second (optimal) phase of the model (1B). The results from each model included the two standard base-case results. In addition, results from each combination of six levels of incomplete coverage $(0 \%, 20 \%, 40 \%, 60 \%, 80 \%$
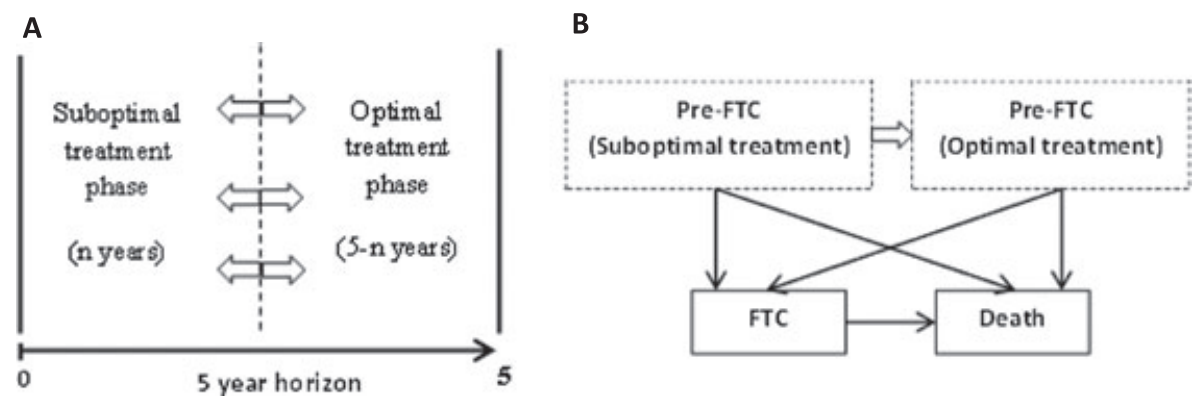

Figure 1 Model timing and schematic. Panel A model time horizon showing demarcation into suboptimal and optimal treatment phases that vary; panel B schematic that shows the transition of patients as disease progresses and movement of patients from suboptimal to optimal treatment. FTC, full-time care. 
and $100 \%$ ) and five different lengths of delay to optimal treatment $(1,2,3,4$ and 5 years) were produced.

As with the original, the model ran for a total of 60 one-month cycles, and so the time horizon was 5 years. Costs were from a National Health Service (NHS) health and social care perspective and are reported in 2016 British pounds. The primary outcome for patients is the quality-adjusted life year (QALY), which is a measure of survival weighted by utilities that capture individual health-related quality of life longitudinal epidemiological study (LASER-AD), a cohort of 224 patients comprising a roughly equal mix of mild, moderate and severe AD dementia, with dementia severity defined by scores on the MiniMental State Examination (Livingston et al., 2004). The majority of patients were taking AChEIs at baseline $(72 \%)$. A discrete version of the Cox proportional hazards model with a complimentary log-log specification was fitted (Rive et al., 2010a; Rive et al., 2010b).

$$
\begin{aligned}
P_{i}^{\mathrm{FTC}}= & 1-\exp \left[-\exp \left(-11.1343+0.0330 * \mathrm{ADAS}-\operatorname{cog}_{\text {baseline }}-0.0877 * \mathrm{ADSC}_{-} \mathrm{ADl}_{\text {baseline }}+0.0377 * \mathrm{NPI}_{\mathrm{baseline}}+0.8122\right.\right. \\
& \left.\left.* \mathrm{ADAS}-\operatorname{cog}_{\text {slope }}-2.4072 * \mathrm{ADSC}-\mathrm{ADl}_{\text {slope }}\right) * \exp (3.3195 * \ln (\text { end month of interval } i))\right]
\end{aligned}
$$

on a scale from 1 (perfect health) to 0 (death) (Sassi, 2006). All costs and outcomes were discounted at $3.5 \%$ per annum.

\section{Data sources}

Disease progression and transition to full-time care. The key advantage of this modelling framework is that the covariates in the equation determining the pre-FTC to FTC transition include all of the well-established AD domains (cognition, function, behaviour), as measured by the total scores on the following clinical scales: $\mathrm{AD}$ Assessment Scale cognitive sub-score (ADAS-cog), AD Cooperative Study Activities of Daily Living scale (ADCS-ADL) and the Neuropsychiatric Inventory (NPI) total score. The equation was fit to data from the London and South-East Region
Fixed covariates include the baseline values of all three scales and the average monthly rates of change (i.e. slopes) of ADAS-cog and ADCS-ADL. Probabilities of movement to FTC varied with interval $i$ and were converted into monthly (per cycle) transition probabilities before being entered into the model.

Baseline scale, slope and treatment effects for the moderate-to-severe model were taken from the original model, and inputting these gave the standard care transition probabilities to FTC (Rive et al., 2010a). Baseline and slope statistics were calculated using moderate-to-severe patients from LASER-AD (Table 1). Baseline statistics for the mild-to-moderate model were taken from the control arm (202 patients with $70 \%$ AChEI use) of a 24-week, double-blind trial comparing memantine with AChEIs in a mild-tomoderate AD population (Peskind et al., 2006). Given

\begin{tabular}{|c|c|c|c|}
\hline \multicolumn{2}{|c|}{ Parameters } & \multirow{2}{*}{$\begin{array}{c}\text { Model 1: moderate-to-severe AD } \\
\text { Mean (SE) }\end{array}$} & \multirow{2}{*}{$\begin{array}{c}\text { Model 2: mild-to-moderate AD } \\
\text { Mean (SE) }\end{array}$} \\
\hline Standard care & ADAS-cog baseline & & \\
\hline & ADCS-ADL baseline & 45.00 & $56.2(0.93)$ \\
\hline & NPI baseline & $18.54(1.86)$ & $12.2(0.92)$ \\
\hline & ADAS-cog slope ${ }^{a}$ & 0.6116 (0.0809) & $0.1667(0.1033)$ \\
\hline & ADCS-ADL slope ${ }^{b}$ & $-0.7503(0.0876)$ & $-0.3833(0.1400)$ \\
\hline \multirow[t]{3}{*}{ Treatment effects } & ADAS-cog ${ }^{a}$ & $-1.54(0.31)$ & $-2.67(1.22)$ \\
\hline & $A D C S-A D^{b}$ & $1.53(0.62)$ & $0.9(0.36)$ \\
\hline & $\mathrm{NPI}^{\mathrm{a}}$ & $-1.34(0.93)$ & $-3.51(0.45)$ \\
\hline
\end{tabular}

Table 1 Predictive equation parameters and treatment effects

AD, Alzheimer's disease; ADAS-cog, AD Assessment Scale cognitive sub-score; ADCS-ADL, AD Cooperative Study Activities of Daily Living scale; NPI, Neuropsychiatric Inventory.

${ }^{a}$ Negative change or treatment effect indicates improvement.

${ }^{\mathrm{b}}$ Positive change or treatment effect indicates improvement. 
that most of the patients were treated with ACHEIs in the LASER-AD cohort that was used to fit the predictive equation, by entering these scale statistics, we obtained time-varying transition probabilities for the optimal treatment arm.

Treatment effects. As in Rive et al. (2010a), the transition probabilities for the other treatment arm were obtained by applying a treatment effect additively to the baseline scale parameters but not to the slopes and so assumed there is no disease modifying effect. This is consistent with previous literature and the original published framework (Green et al., 2005; Green, 2007; Cohen and Neumann, 2008; Rive et al., 2010a; Rive et al., 2010b). For the moderate-to-severe $\mathrm{AD}$ cohort, treatment effects were weighted mean differences taken from a meta-analysis of 6-month randomised controlled trials most of which compared memantine monotherapy to AChEIs (Rive et al., 2010a; Winblad et al., 2007).

For the mild-to-moderate cohort, meta-analyses for obtaining treatment effects were sought from the published literature in order to satisfy the following conditions: treatment effects combined were around 24 weeks; most of the patients in the combined studies were mild-to-moderate severity; treatment was donepezil, and treatment effects were on their original scales (weighted mean differences). The treatment effect for donepezil on ADAS-cog was taken from a meta-analysis of mainly 6-month randomised controlled trials that compared AChEIs in mild-tomoderate $\mathrm{AD}$ patients (Hansen et al., 2008). The treatment effect for donepezil on the NPI was taken from a similar meta-analysis of four trials (Lockhart et al., 2011). No recently published meta-analyses (Hansen et al., 2008; Lockhart et al., 2011; Di Santo et al., 2013; Kobayashi et al., 2015) or trials within these satisfied the conditions for a treatment effect for donepezil on the ADCS-ADL. Therefore, a treatment effect for galantamine from a large 5-month trial was inputted for this scale instead (Tariot et al., 2000). These treatment effects were then 'reversed' (placebo versus AChEI) before being additively applied to scale baselines to produce the transition probabilities for the standard care arm.

Switching to optimal treatment. Depending on the scenario, there will be some patients who are in that proportion of the modelled cohort with treatment coverage and experiencing no delays. These receive the NICE recommended treatment from the beginning and are unaffected by the change in phase; they transition for the 5 years with treatment effects applied from time 0 . When patients starting suboptimal treatment reached the second phase and switched to optimal treatment, the assumption was that they continue in the same way as those who received optimal treatment from the beginningprognosis is independent of previous experience.

Two other assumptions were evaluated during sensitivity analyses. First, at point of switching, treatment effects were applied to baseline scales that had been updated using the monthly rates of change (slopes), and the time variable in the predictive equation was reset to zero. This did not capture the disease progression that occurs independent of any treatment effect and that is so important in modelling $\mathrm{AD}$. In fact, this approach actively 'punished' patients in terms of QALYs and time in pre-FTC for having the optimal treatment from the beginning. Second, treatment effects were again applied to updated baseline scales, but the time variable was not reset. This also implied unrealistic disease progression; over 5 years, switchers were worse off in QALY and time in pre-FTC terms relative to people who received suboptimal treatment from time 0 (standard care arm).

Mortality. As in the original Rive et al. (2010a) model, the second transition patients can experience-from pre-FTC or FTC to death-was determined by a survival analysis using a Weibull parameterisation. The assumption of no treatment effect on mortality was maintained. The full LASER-AD cohort not divided by severity was used to fit the equation, and so probabilities of death do not vary by severity model.

Costs. For both models, the mean (SE) monthly costs of care for pre-FTC and FTC were $£ 805$ ( $£ 217)$ and $£ 3634$ ( 2284 ), respectively. The resources used for these states were collected from the LASER-AD study. In each model, monitoring costs associated with the optimal treatments were applied: initial consultation with a specialist at the start of treatment ( $£ 139)$ followed by general practitioner visits every half year

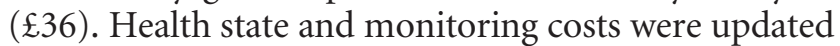
or adjusted using the hospital and community health services index to reflect 2016 prices (Curtis, 2016). The daily cost of memantine was inputted as $\mathfrak{E} 0.053$. The assumption that treatments stop when patients enter FTC or death is also continued. For the mildto-moderate model, the daily cost of donepezil was inputted ( $£ 0.055)$ because this is the most widely prescribed AChEI in the UK (Cooper et al., 2016). These drug costs account for the recent expiration of patents and reflect the current daily costs of these drugs as listed in the British National Formulary 
(BNF, 2016). They are substantially lower than previously reported drug costs: memantine at $£ 2.16$ (Rive et al., 2010a) and donepezil at $\mathfrak{E 3 . 1 8}$ per day (Getsios et al., 2012). No treatment cost was incurred in standard care in either severity model because it is background therapy for all patients and so makes no difference to incremental costs.

Outcomes. As in the original model, pre-FTC (EQ$5 \mathrm{D})$ utilities were time varying and determined by a generalised linear model fitted to the LASER-AD cohort data (Rive et al., 2010a). As with the predictive equation, treatment effects were applied to baselines and the same assumption about patients that switch was made. The utilities in the FTC state for moderate-to-severe and mild-to-moderate models were means calculated from patient cohorts in FTC with the relevant severity AD: 0.336 and 0.340 , respectively (Rive et al., 2010a; Caro et al., 2002).

Net monetary benefit (NMB) is the monetary value of average QALY benefits net of the average costs of treatment, assuming the willingness-to-pay for a QALY is the NICE specified $\mathfrak{E 3 0}$ 000: $\mathrm{NMB}=$ QALYs $\times \mathfrak{E 3 0} 000-$ costs. Incremental NMB was calculated for optimal care and each scenario as the difference in NMB relative to the NMB for (base-case) standard care.

\section{Results}

\section{Base-case results}

Table 2 shows base-case deterministic results and the uncertainty around these estimates in terms of standard errors from the probabilistic sensitivity analysis. The optimal treatment in both models dominates standard care-average patient costs are lower and QALYs higher over 5 years. When the costs inputted were identical to the original publication (Rive et al., 2010a), results in the moderate-to-severe model were also the same. However, with the updated (and higher) input costs, total costs increase in both arms. The difference in costs also increases because of the drop in the price of memantine.

When the previous price of donepezil was inputted, the results for the mild-to-moderate model were similar to the results produced by the models that informed NICE guidance for mild-to-moderate $\mathrm{AD}$ (NICE, 2011; Bond et al., 2012): AChEI therapy saved around $\mathfrak{E} 600$ and increased QALYs by 0.035 . With updated costs, AChEI therapy continues to dominate standard care, but cost savings are substantially higher.

\section{Moderate-to-severe scenario results}

Figures 2 and 3 show the results for all coverage-delay scenarios (see Appendix A of the Supporting Information). The dashed lines represent the fixed base-case results from Table 2. The relationship between delay and outcomes, with fixed coverage, is non-linear. This is because the predictive equation moves patients to FTC at a non-linear rate, and so the marginal effect of delay on outcomes will vary by level of delay. Coverage has a linear relationship with all outcomes, which can be seen by noting the equal spacing between curves; indeed, when the horizontal axis is coverage instead of delay, the figures yield straight lines. Changing coverage has an additive impact because it involves swapping patients between defined pathways. As coverage increases or delay decreases, beyond a year outcome values tend towards the base-case optimal value.

For the moderate-to-severe cohort, optimal treatment (memantine) for all patients from the beginning provides lowest costs and highest benefits. Delaying treatment increases costs and decreases

Table 2 Five-year (per patient) base-case results

\begin{tabular}{lccc}
\hline & Standard care & Optimal & Difference \\
\hline Model 1: moderate to severe ${ }^{\mathrm{a}}$ (mean \pm SE) & & & \\
Total costs $(£)$ & $£ 105420 \pm 9346$ & $£ 102020 \pm 9313$ & $-£ 3400 \pm 1121$ \\
QALYs & $1.502 \pm 0.092$ & $1.533 \pm 0.089$ & $0.031 \pm 0.013$ \\
Time in pre-FTC (months) & $19.8 \pm 1.6$ & $21.1 \pm 1.7$ & $1.3 \pm 0.4$ \\
Model 2: mild to moderate (mean \pm SE) & $£ 62988 \pm 6170$ & $£ 58545 \pm 5599$ & $-£ 4443 \pm 1030$ \\
Total costs (£) & $1.958 \pm 0.108$ & $2.004 \pm 0.110$ & $0.047 \pm 0.014$ \\
QALYs & $34.8 \pm 2.2$ & $36.5 \pm 2.0$ & $1.7 \pm 0.4$ \\
Time in pre-FTC (months) & & & \\
\end{tabular}

QALY, quality-adjusted life years; FTC, full-time care.

${ }^{a}$ The deterministic and probabilistic results are virtually identical with the Rive et al. (2010a) model when cost inputs are the same. The results presented here are shown for the updated cost inputs and drug costs that reflect modern prices. 


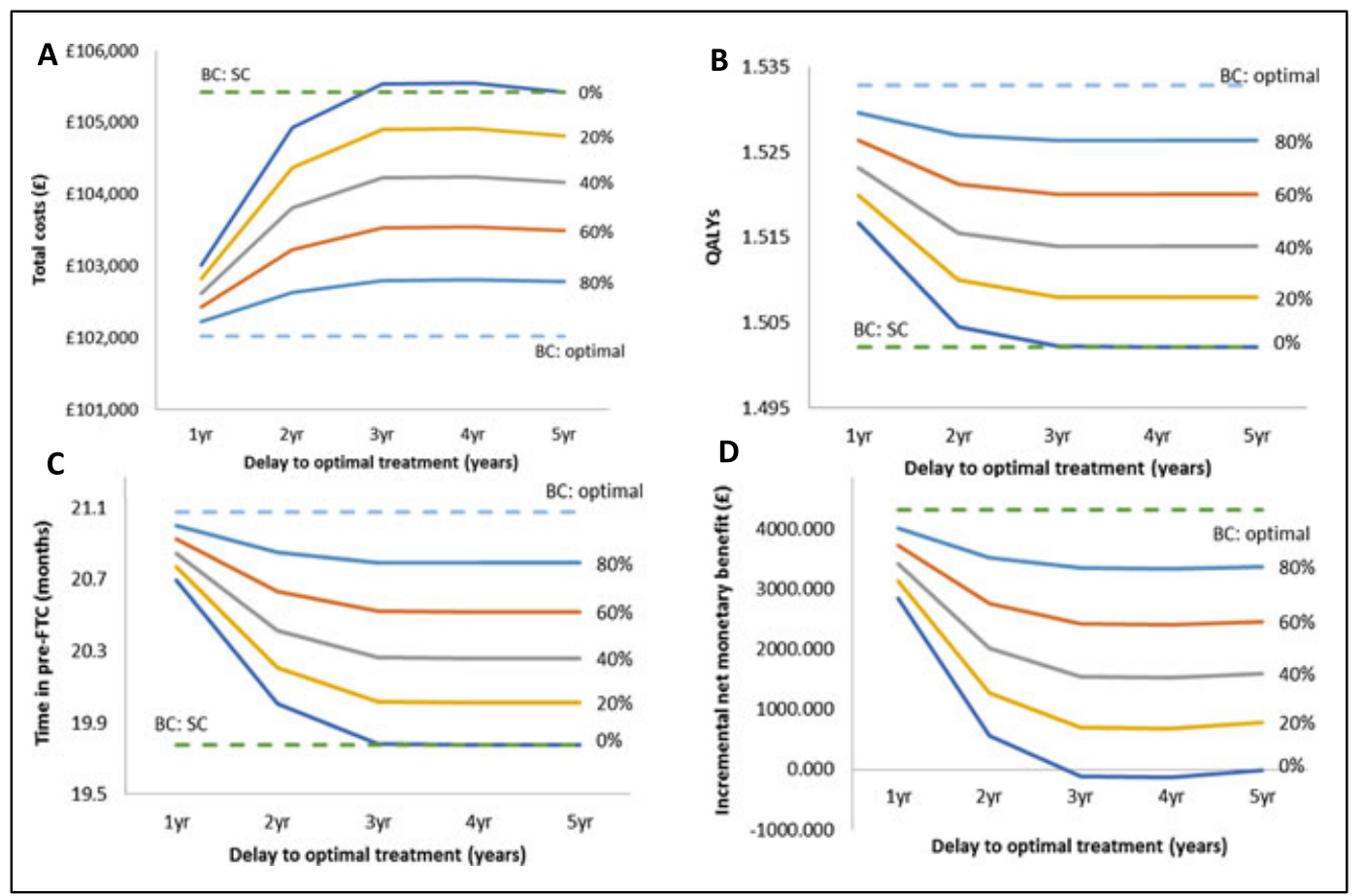

Figure 2 Moderate-to-severe model (per patient) results: coverage and delay in treatment scenarios. Panel A average total costs over 5 years; panel B average quality-adjusted life years (QALYs); panel C average time (months) spent in pre-full-time care (FTC) state; panel D average incremental net monetary benefit relative to base-case (BC) standard care (SC). [Colour figure can be viewed at wileyonlinelibrary.com]

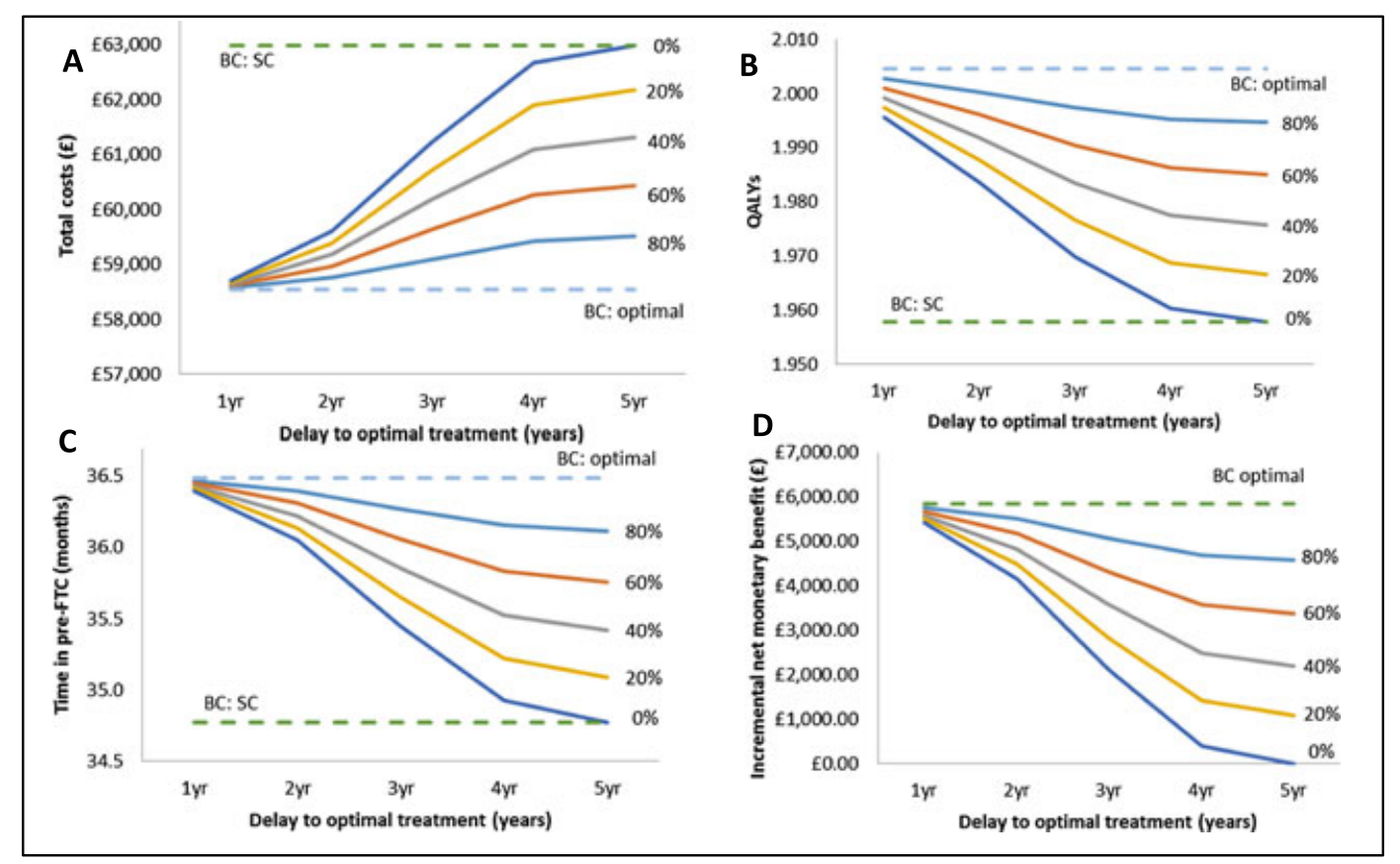

Figure 3 Mild-to-moderate model (per patient) results: coverage and delay in treatment scenarios. Panel A average total costs over 5 years; panel B average quality-adjusted life years (QALYs); panel C average time (months) spent in pre-full-time care (FTC) state; panel D average incremental net monetary benefit relative to base-case (BC) standard care (SC). [Colour figure can be viewed at wileyonlinelibrary.com] 
benefits but at a decreasing rate. Leaving patients without optimal treatment also increases costs and decreases benefits.

Published estimates place untreated $\mathrm{AD}$ at around 50\% (Getsios et al., 2012; National Audit Office, 2007) and average delays to diagnosis at 3 years (Bond et al., 2005). Assuming this as the status quo, pursuing a strategy of early diagnosis with all patients receiving optimal treatment immediately, we would expect an average saving of up to $\mathfrak{E} 1856$ over 5 years. We would also expect average NMB to increase by up to $\mathfrak{E} 1893$. As another example, if delay was 3 years for the typical patient and a programme increased coverage from $50 \%$ to $55 \%$, incremental $\mathrm{NMB}$ would increase by $\mathfrak{E} 175$. This would be an upper limit; an average cost figure associated with the programme, true negative cases and false positive cases would have to be subtracted.

\section{Mild-to-moderate scenario results}

For the mild-to-moderate cohort, all results are similar to the moderate-to-severe cohort-average benefits decrease and costs increase with delays to treatment (AChEIs) and proportion left untreated (3B and 3C). Relative to the moderate-to-severe model, these outcomes changed more slowly and did not level off with later delays. Assuming that at present $50 \%$ of cases go undiagnosed and average delay is 3 years, if all patients were to receive treatment without delay, we would expect cost savings of up to $£ 1375$ and an increase in NMB of around $£ 1902$.

When the older price for donepezil is inputted, coverage-delay scenarios are drastically different (see Appendix B of the Supporting Information). Increasing coverage is actually punished in cost terms for delays up to around 3 years, and after this turning point, the relationship between coverage and cost is reversed. Any proportion of patients left untreated for delays to AChEI treatment up to 3 years will yield lower average costs than the scenario where all patient receive AChEI treatment from time 0 . The most costeffective strategy was a delay in AChEI treatment for everyone for some point up to 1 year. Inputting the older price for memantine did not change the results of the moderate-to-severe model a great deal.

\section{Discussion}

This study aimed to investigate how changes in delay, coverage and disease severity determine the costeffectiveness of pharmacological treatments for $\mathrm{AD}$. Cost results are different for each severity cohort, despite treatment costs being very similar and monitoring, pre-FTC and FTC input costs being identical. Total costs are a balance between two processes: direct treatment (drug and monitoring) costs increase with lower delays and higher coverage, but these increases are offset by lower health state costs as patients move more slowly into expensive FTC. The balance between these processes is determined by the equation that predicts movement to FTC, and it predicts very different rates of movement over time for each cohort (Appendix C of the Supporting Information).

Getsios et al. (2012) evaluated early assessment in the UK for suspected mild-to-moderate $\mathrm{AD}$, defined as more frequent reassessment within the current diagnostic pathway. In the Getsios et al. base-case, the time to diagnosis (and donepezil treatment) was set at 36 months in the latter strategy. Both Figure 3 and the analysis in which the price of donepezil is set to the higher price reported in Getsios et al. (Appendix B of the Supporting Information) suggest that when starting from a delay of 3 years (at any coverage), there are gains in cost-effectiveness from any combination of decrease in delay and increase in coverage. Therefore, this study is consistent with previous work: early assessment-as defined in Getsios et al.increases cost-effectiveness.

The main limitation of the framework-as with almost all models in $\mathrm{AD}$-was the assumption that the (6-month) treatment effects were maintained over the model duration. The LASER-AD cohort was composed of only patients residing in urban areas, and so there may be some issue with generalising the results to the entire UK population. In the moderateto-severe model, both the original model publication (Rive et al., 2010a) and this study are suggesting that memantine monotherapy is the active treatment being modelled. There is some concern that the applied treatment effect may be biased because two of the six trials used in the relevant meta-analysis (Winblad et al., 2007) included background treatment with donepezil. However, there is little clinical evidence that monotherapy and combination therapy differ in efficacy (NICE, 2011). A more general criticism is that other modelling approaches (e.g. discrete event simulation) are better at capturing the heterogeneous nature of $\mathrm{AD}$ progression. However, the use of timevarying probabilities (and utilities) based on predictive equations fit to individual-level data can compensate for the disadvantages of Markov modelling.

More caution needs to be taken in interpreting the outputs for the mild-to-moderate $\mathrm{AD}$ model. First, the LASER-AD cohort contained AD patients of all severities, but the fitted equation was used separately 
in the two differing severity cohorts to predict movement to FTC. Second, the modelled trial cohort (Peskind et al., 2006) was based in the USA and may not be entirely representative of the UK population (Herrmann et al., 2015; Dodel et al., 2015). Third, the cohort used to fit the predictive equation (LASER-AD) is different from this modelled cohort, unlike in the moderate-to-severe analysis. Fourth, the calculated scale slopes were based on 24-week change, which may misrepresent disease progression. However, as suggested, the results for the mild-to-moderate model are in line with previous work.

In final conclusion, QALYs would be increased in both severity groups by programmes designed to improve coverage and reduce delay to optimal treatment. Such programmes and the related technology (Blackwell et al., 2004; IXICO, 2014; Heister et al., 2011) also have good potential to be cost saving by reducing delay to NICE recommended treatment. This conclusion has been strengthened by the recent expiration of patents and subsequent low cost of approved pharmacological treatments for $\mathrm{AD}$ in the UK.

\section{Ethics statement}

Ethical approval was not required. This study did not involve research on human subjects, and so no ethics approval was sought.

\section{Conflict of interest}

None declared.

\section{Key points}

- The suboptimal pharmacological treatment of $\mathrm{AD}$ in the UK can be defined in terms of low coverage (percentage of total patient population receiving treatment) and excessive delay to treatment.

- Quality of life was maximised and costs minimised in both severity groups (mild-tomoderate and moderate-to-severe $\mathrm{AD}$ ) when delay was minimised and coverage maximised.

- For the mild-to-moderate $\mathrm{AD}$ group, these results were highly sensitive to the inputted cost of approved treatment (AChEIs).

- When the previous (higher) price of donepezil was used for the mild-to-moderate $\mathrm{AD}$ group, cost-effectiveness was no longer maximised by minimising delay and maximising coverage.

\section{Acknowledgement}

This work was supported by Innovate UK.

\section{References}

Alzheimers Association. 2011. Alzheimer's disease facts and figures. Alzheimers Dement 7: 208-244 https://www.alz.org/downloads/Facts_Figures_2011.pdf.

Banerjee S, Wittenberg R. 2009. Clinical and cost effectiveness of services for early diagnosis and intervention in dementia. Int $J$ Geriatr Psych 24: $748-754$.

Barnett JH, Lewis L, Blackwell AD, Taylor M. 2014. Early intervention in Alzheimer's disease: a health economic study of the effects of diagnostic timing. BMC Neurol 14: 101 .

Blackwell AD, Sahakian BJ, Vesey R, et al. 2004. Detecting dementia: novel neuropsychological markers of preclinical Alzheimer's disease. Dement Geriatr Cogn Disord 17: 42-48.

Bond J, Stave C, Sganga A, O'Connell B, Stanley RL. 2005. Inequalities in dementia care across Europe: key findings of the Facing Dementia Survey. Int J Clin Pract 59(Suppl 146): 8e14.

Bond M, Rogers RG, Peters J, et al. 2012. The effectiveness and cost-effectiveness of donepezil, galantamine, rivastigmine and memantine for the treatment of Alzheimer's disease (review of Technology Appraisal No. 111): a systematic review and economic model. Health Technol Assess 16(21): 1-470.

British National Formulary. 2016. https://www.medicinescomplete.com/mc/bnf/ current/.

Caro JJ, Salas M, Ward A, Getsios D, Mehnert A. 2002. Economic analysis of galantamine, a cholinesterase inhibitor, in the treatment of patients with mild to moderate Alzheimer's disease in the Netherlands. Dement Geriatr Cogn Disord 14(2): 84-89.

Cohen J, Neumann P. 2008. Decision analytic models for Alzheimer's disease: state of the art and future directions. Alzheimers Dement 4: 212-222.

Cooper C, Lodwick R, Walters K, et al. 2016. Observational cohort study: deprivation and access to anti-dementia drugs in the UK. Age Ageing 45(1): 148-154.

Curtis L. 2016. PSSRU: unit costs of health and social care.

Di Santo SG, Prinelli F, Adorni F, Caltagirone C, Musicco M. 2013. A meta-analysis of the efficacy of donepezil, rivastigmine, galantamine, and memantine in relation to severity of Alzheimer's disease. J Alzheimers Dis 35(2): 349-361.

Dodel R, Belger M, Reed C, et al. 2015. Determinants of societal costs in Alzheimer's disease: GERAS study baseline results. Alzheimers Dement 11(80): 933-945.

Getsios D, Blume S, Ishak KJ, Maclaine G, Hernández L. 2012. An economic evaluation of early assessment for Alzheimer's disease in the United Kingdom. Alzheimers Dement 8: 22-30.

Green C, Picot J, Loveman E, et al. 2005. Modelling the cost effectiveness of cholinesterase inhibitors in the management of mild to moderately severe Alzheimer's disease. Pharmacoeconomics 23: 1271-1282.

Green C. 2007. Modelling disease progression in Alzheimer's disease: a review of modelling methods used for cost-effectiveness analysis. Pharmacoeconomics 25: 735-750.

Hansen RA, Gartlehner G, Webb AP, et al. 2008. Efficacy and safety of donepezil, galantamine, and rivastigmine for the treatment of Alzheimer's disease: a systematic review and meta-analysis. Clin Interv Aging 3(2): 211-225.

Heister D, Brewer JB, Magda S, Blennow K, McEvoy LK. 2011. Predicting MCI outcome with clinically available MRI and CSF biomarkers. Neurology 77(17): 1619-1628.

Herrmann N, Harimoto T, Balshaw R, Lanctôt KL. 2015. Risk factors for progression of Alzheimer disease in a Canadian population: the Canadian Outcomes Study in Dementia (COSID). Canadian Journal of Psychiatry Revue Canadienne de Psychiatrie 60(4): 189-199 https://www.nice.org.uk/guidance/ta217.

Kobayashi H, Ohnishi T, Nakagawa R, Yoshizawa K. 2015. The comparative efficacy and safety of cholinesterase inhibitors in patients with mild-to-moderate Alzheimer's disease: a Bayesian network meta-analysis. Int J Geriatr Psychiatry 31(8): 892-904.

Livingston G, Katona C, Roch B, Rive B. 2004. A dependency model for patients with Alzheimer's disease: its validation and relationship to the costs of care-the LASER-AD Study. Curr Med Res Opin 20: 1007-1016.

Lockhart IA, Orme ME, Mitchell SA. 2011. The efficacy of licensed-indication use of donepezil and memantine monotherapies for treating behavioural and psychological symptoms of dementia in patients with Alzheimer's disease: systematic review and meta-analysis. Dement Geriatr Cogn Dis Extra 1(1): 212-227.

National Audit Office. 2007. Improving services and support for people with dementia. London: The Stationery Office. https://www.nao.org.uk/wp-content/ uploads/2007/07/0607604.pdf 
National Institute for Health and Care Excellence, Dementia diagnosis and assessment. 2016. NICE pathways. https://pathways.nice.org.uk/pathways/ dementia/dementia-diagnosis-and-assessment.

National Institute for Health and Care Excellence. 2011. Donepezil, galantamine, rivastigmine and memantine for the treatment of Alzheimer's disease. Technology appraisal guidance.

Peskind ER, Potkin SG, Pomara N, et al. 2006. Memantine treatment in mild to moderate Alzheimer disease: a 24-week randomized controlled trial. Am I Geriatr Psychiatry 14(8): 704-715.

Prince M, Knapp M, Guerchet M, McCrone P, Prina M, Comas-Herrera A, Wittenberg R, Adelaja B, Hu B, King D, Rehill A, Salimkumar D. 2014. Dementia UK: update. London: Alzheimer's Society, Gordon House. https://www. alzheimers.org.uk/dementiauk.

Rive B, Grishchenko M, Guilhaume-Goulant C, et al. 2010a. Cost effectiveness of memantine in Alzheimer's disease in the UK. J Med Econ 13(2): 371-380.

Rive B, Le Reun C, Grishchenko M, et al. 2010b. Predicting time to full-time care in AD: a new model. J Med Econ 13: 362-370.

Sassi F. 2006. Calculating QALYs, comparing QALY and DALY calculations. Health Policy Plan 21(5): 402-408.

Sonnenberg FA, Beck JR. 1993. Markov models in medical decision making: a practical guide. Med Decis Making 13(4): 322-338.

Tariot PN, Solomon PR, Morris JC, et al. 2000. A 5-month, randomized, placebocontrolled trial of galantamine in AD. The Galantamine USA-10 Study Group. Neurology 54(12): 2269-2276.

IXICO. 2014 press release: From iPad to Pod—cutting the wait in dementia and brain health assessment http://www.thebrainhealthcentre.com/TBHC_Press_release_912-14.pdf

Weimer DL, Sager MA. 2009. Early identification and treatment of Alzheimer's disease: social and fiscal outcomes. Alzheimers Dement 5: 215-226.

Winblad B, Jones RW, Wirth Y, Stöffler A, Möbius HJ. 2007. Memantine in moderate to severe Alzheimer's disease: a meta-analysis of randomised clinical trials. Dement Geriatr Cogn Disord 24: 20-27.

\section{Supporting Information}

Additional Supporting Information may be found online in the supporting information tab for this article:
Table A.1. Results for all coverage-delay scenarios and both severities

Figure B.1. Moderate-to-severe model (per patient) results with previous drug price: coverage and delay in treatment scenarios with Memantine at $£ 2.16$ per day. Panel A average total costs over 5 years; Panel B average QALYs; Panel C average time (months) spent in pre-FTC state; Panel D average incremental net monetary benefit relative to $\mathrm{BC}$ standard care ( $\mathrm{BC}=$ base-case, $\mathrm{SC}=$ standard care $)$

Figure B.2. Mild-to-moderate model (per patient) results with previous drug price: coverage and delay

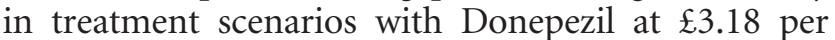
day. Panel A average total costs over 5 years; Panel B average QALYs; Panel C average time (months) spent in pre-FTC state; Panel D average incremental net monetary benefit relative to $\mathrm{BC}$ standard care $(\mathrm{BC}=$ base-case, $\mathrm{SC}=$ standard care $)$

Figure C.1. Probability of movement to FTC for moderate-to-severe $\mathrm{AD}$ patients

Figure C.2. Probability of movement to FTC for mildto-moderate $\mathrm{AD}$ patients

Figure C.3. Proportion of patient cohort in pre-FTC (moderate-to-severe AD patients)

Figure C.4. Proportion of patient cohort in pre-FTC (mild-to-moderate AD patients) 